\title{
Comparative study of an Eden model for the irreversible growth of spins and the equilibrium Ising model
}

\author{
Julián Candia* and Ezequiel V. Albano ${ }^{\dagger}$ \\ Instituto de Investigaciones Fisicoquímicas Teóricas y Aplicadas (INIFTA), CONICET, Universidad Nacional de La Plata, CIC (Bs. As.), \\ Sucursal 4, Casilla de Correo 16, (1900) La Plata, Argentina \\ (Received 4 October 2000; revised manuscript received 28 February 2001; published 25 May 2001)
}

\begin{abstract}
The magnetic Eden model (MEM) [N. Vandewalle and M. Ausloos, Phys. Rev. E 50, R635 (1994)] with ferromagnetic interactions between nearest-neighbor spins is studied in $(d+1)$-dimensional rectangular geometries for $d=1,2$. In the MEM, magnetic clusters are grown by adding spins at the boundaries of the clusters. The orientation of the added spins depends on both the energetic interaction with already deposited spins and the temperature, through a Boltzmann factor. A numerical Monte Carlo investigation of the MEM has been performed and the results of the simulations have been analyzed using finite-size scaling arguments. As in the case of the Ising model, the MEM in $d=1$ is noncritical (only exhibits an ordered phase at $T=0$ ). In $d=2$ the MEM exhibits an order-disorder transition of second order at a finite temperature. Such transition has been characterized in detail and the relevant critical exponents have been determined. These exponents are in agreement (within error bars) with those of the Ising model in two dimensions. Further similarities between both models have been found by evaluating the probability distribution of the order parameter, the magnetization, and the susceptibility. Results obtained by means of extensive computer simulations allow us to put forward a conjecture that establishes a nontrivial correspondence between the MEM for the irreversible growth of spins and the equilibrium Ising model. This conjecture is certainly a theoretical challenge and its confirmation will contribute to the development of a framework for the study of irreversible growth processes.
\end{abstract}

DOI: 10.1103/PhysRevE.63.066127

PACS number(s): 64.60.Cn, 05.40.-a, 81.15.Aa, 05.50.+q

\section{INTRODUCTION}

The study of kinetic growth models such as directed percolation, Eden growth, ballistic deposition, diffusion limited aggregation, random deposition with and without relaxation, cluster-cluster aggregation, etc., is motivated by their interest in many areas of scientific research and technology such as polymer science, crystal and polycrystalline growth, gelation, fracture propagation, epidemic spreading, bacterial and fungi growth colonies, colloids, etc. [1-5]. Within this context the Eden model [6] has become an archetype growth model. Eden clusters are compact but the self-affinity that characterizes the behavior of the growing interface is of much interest (see, e.g., [7-12]). Few years ago Ausloos et al. [13] have introduced an additional degree of freedom to the Eden model, namely, the spin of the added particles. More recently, the Eden growth of clusters of charged particles has also been studied [14].

In the magnetic Eden model (MEM) [13] with spins having two orientations (up and down) the growth of the cluster starts from a single seed, e.g., a spin up seed, placed at the center of the two-dimensional square lattice, whose sites are labeled by their rectangular coordinates $(i, j)$. Then, the growth process of the resulting magnetic cluster consists in adding further spins to the growing cluster taking into ac-

\footnotetext{
*Present and Permanent address: Departamento de Física, Facultad de Ciencias Exactas, Universidad Nacional de La Plata, CC 67, (1900) La Plata, Argentina.

†FAX: 0054-221-4254642.

Email address: ealbano@inifta.unlp.edu.ar
}

count the corresponding interaction energies. By analogy to the Ising model [15] one takes $J$ as the coupling constant between nearest-neighbor (NN) spins $S_{i j}$ and the energy $E$ is then given by

$$
E=-\frac{J}{2} \sum_{\left\langle i j, i^{\prime} j^{\prime}\right\rangle} S_{i j} S_{i^{\prime} j^{\prime}},
$$

where $\left\langle i j, i^{\prime} j^{\prime}\right\rangle$ means that the summation is taken over occupied NN sites. The spins can assume two values, namely, $S_{i j}= \pm 1$. Throughout this work we set the Boltzmann constant equal to unity $\left(k_{B} \equiv 1\right)$, we consider $J>0$ (i.e., the ferromagnetic case) and we take the absolute temperature $T$ measured in units of $J$. In the MEM a spin is added to the cluster with a probability proportional to the Boltzmann factor $\exp (-\Delta E / T)$, where $\Delta E$ is the total energy change involved. It should be noted that at each step all sites of the perimeter are considered and the probabilities of adding up and down spins have to be evaluated. After proper normalization of the probabilities the growing site and the orientation of the spin are determined through a pseudorandom number generator.

It is worth mentioning that the MEM has originally been motivated by the study of the structural properties of magnetically textured materials [13]. While these previous studies of the MEM were mainly devoted to determine the lacunarity exponent and the fractal dimension of the set of parallel oriented spins [13], the aim of the present work is to complement these previous investigations by studying the critical behavior of the MEM using extensive Monte Carlo simulations and applying a finite-size scaling theory. Also, our study is performed in confined (stripped) geometries that resemble recent experiments where the growth of quasi-one- 
dimensional strips of $\mathrm{Fe}$ on a $\mathrm{Cu}(111)$ vicinal surface [16] and $\mathrm{Fe}$ on a W(110) stepped substratum [17] have been performed. In fact, the preparation and characterization of magnetized nanowires is of great interest for the development of advanced microelectronic devices [16-19]. Furthermore, the growth of metallic multilayers of $\mathrm{Ni}$ and Co separated by a $\mathrm{Cu}$ spacer layer has recently been also studied [20].

Another goal of the present work is to compare the results obtained for the MEM with the well-known behavior of the classical Ising model $[15,21]$, an archetypical model in the study of thermally driven (reversible) phase transitions in equilibrium systems. The Ising Hamiltonian $(\mathcal{H})$ is given by

$$
\mathcal{H}=-\frac{J}{2} \sum_{\left\langle i j, i^{\prime} j^{\prime}\right\rangle} S_{i j} S_{i^{\prime} j^{\prime}}
$$

where $\left\langle i j, i^{\prime} j^{\prime}\right\rangle$ means that the summation runs over all NN sites, $S_{i j}= \pm 1$ is the state of the spin at the site of coordinates $(i, j)$ and $J$ is the coupling constant $(J>0)$.

The MEM is also similar to a family of models for the stochastic growth of crystals generically known as crystal growth models (CGM) [22-26], for a review, see e.g., [27]. As in the MEM, in the case of CGM each atom is adsorbed with a given probability conditional to the actual configuration of neighboring atoms on the previous layer(s). However, in contrast to the MEM, the crystal is supposed to grow layer after layer. It should also be noticed that relationships established between CGM and a special class of Ising models $[24,26,28]$ have allowed to derive exact results. Therefore, useful comparisons with the MEM will be also discussed in the presentation of our results.

This paper is organized as follows. In Sec. II we give details on the simulation method, Sec. III is devoted to the presentation and discussion of the results obtained for the MEM in $(1+1)$-dimensions, while Sec. IV refers to results corresponding to $(2+1)$-dimensions. In Secs. III and IV, detailed discussions comparing our results with the behavior of the Ising magnet are outlined. Finally our conclusions are stated in Sec. V.

\section{DESCRIPTION OF THE SIMULATION METHOD}

The MEM in $(1+1)$-dimensions is studied in the square lattice using a rectangular geometry $L \times M$ with $M \gg L$ and imposing periodic boundary conditions along the $L$ direction. The location of each site on the lattice is specified through its rectangular coordinates $(i, j),(1 \leqslant i \leqslant M, 1 \leqslant j \leqslant L)$. The starting seed for the growing cluster is a column of parallel oriented spins placed at $i=1$. It should be noticed that previous simulations of the MEM were restricted to rather modest cluster sizes, i.e., containing up to 8000 spins [13], while in the present work clusters having up to $10^{9}$ spins have been typically grown. We have also studied the MEM in $(2+1)$-dimensions employing an $L \times L \times M$ geometry ( $M$ $\gg L$ ) with periodic boundary conditions along both $L$ directions.

\section{STUDY OF THE MEM IN (1+1)-DIMENSIONS: RESULTS AND DISCUSSION}

Magnetic Eden clusters grown on a stripped geometry of finite linear dimension $L$ at low temperatures show an interesting behavior that we call magnetization reversal. In fact, we have observed that long clusters are constituted by a sequence of well-ordered magnetic domains. Spins belonging to each domain, of average length $l_{D} \gg L$, have mostly the same orientation and consecutive domains have opposite orientation. Let $l_{R}$ be the characteristic length for the occurrence of the magnetization reversal. Since $l_{R} \sim L$, we then conclude that the problem has two characteristic length scales, namely, $l_{D}$ and $l_{R}$ such that $l_{D} \gg l_{R}$.

In ordinary thermally driven phase transitions, the system changes from a disordered state at high temperatures to a spontaneously ordered state at temperatures below some critical value $T_{c}$ where a second-order phase transition takes place. Regarding the Ising model, one has that, in the absence of an external magnetic field $(H=0)$, the low temperature ordered phase is a state with nonvanishing spontaneous magnetization $\left( \pm M_{s p}\right)$. This spontaneous symmetry breaking is possible in the thermodynamic limit only. In fact, it is found that the magnetization $M$ of a finite sample can pass with a finite probability from a value near $+M_{s p}$ to another near $-M_{s p}$, as well as in the opposite direction. Consequently, the magnetization of a finite system, averaged over a sufficiently large observation time, vanishes at every positive temperature. The equation $M(T, H=0) \approx 0$ holds if the observation time $\left(t_{o b s}\right)$ becomes larger than the ergodic time $\left(t_{\text {erg }}\right)$, which is defined as the time needed to observe the system passing from $\pm M_{s p}$ to $\mp M_{s p}$. Increasing the size of the sample the ergodic time increases too, such that in the thermodynamic limit ergodicity is broken due to the divergence of the ergodic time, yielding broken symmetry. Since Monte Carlo simulations are restricted to finite samples, the standard procedure to avoid the problems treated in the foregoing discussion is to consider the absolute magnetization as an order parameter [29]. Turning back to the MEM, we find that the phenomenon of magnetization reversal also causes the magnetization of the whole cluster to vanish at every nonzero temperature, provided that the length of the cluster $l_{C}$ (which plays the role of $t_{o b s}$ ) is much larger than $l_{D}$ (which plays the role of $t_{e r g}$ ). Therefore, we have measured the mean absolute column magnetization, given by

$$
|m(i, L, T)|=\frac{1}{L}\left|\sum_{j=1}^{L} S_{i j}\right| .
$$

In the stripped geometry used in this work the bias introduced by the lineal seed (a starting column made up entirely of up spins) can be avoided by calculating relevant properties after disregarding spins within a distance approximately equal to few times $L$ from the seed. The procedure of column averaging out from the transient region represents a significant advantage of the stripped geometry used for the simulation of the MEM. In fact, when a single seed at the center of the sample is used, the definition of the average magnetization of the whole cluster is strongly biased by the cluster's 


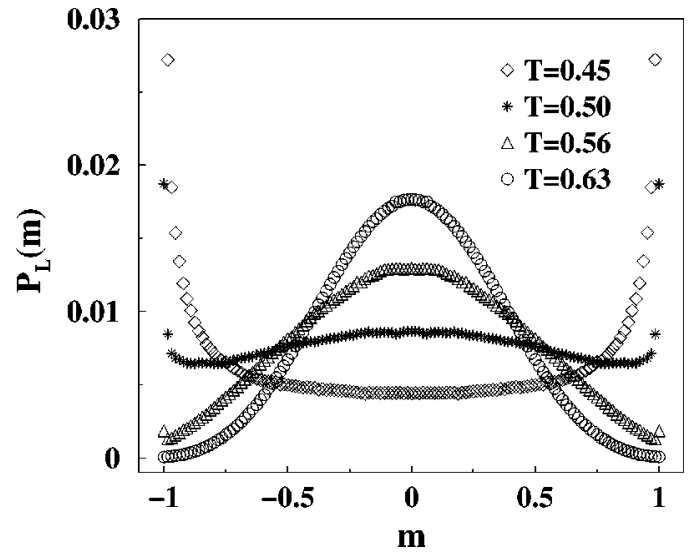

FIG. 1. Plots of the probability distribution of the mean column magnetization $P_{L}(m)$ versus $m$ for the fixed lattice width $L=128$ and different temperatures. The sharp peaks at $m= \pm 1$ for $T$ $=0.45$ have been truncated in order to allow a detailed observation of the plots corresponding to higher temperatures. This behavior resembles that of the one-dimensional Ising model. More details in the text.

kernel orientation at the early stages of the growing process. In addition, using several randomly generated seeds we could also establish that the system evolves into a given stationary state independently of the seed employed.

The mean column magnetization is a fluctuating quantity that can assume $L+1$ values. Then, for given values of both $L$ and $T$, the probability distribution of the mean column magnetization $\left(P_{L}(m)\right)$ can be evaluated, since it represents the normalized histogram of $m$ taken over a sufficiently large number of columns in the stationary region [30-32]. In the thermodynamic limit the probability distribution $\left(P_{\infty}(m)\right)$ of the order parameter of an equilibrium system at criticality is universal (up to rescaling of the order parameter) and thus it contains very useful and interesting information on the universality class of the system [33-35]. Figure 1 shows the thermal dependence of $P_{L}(m)$ for a fixed lattice size ( $L$ $=128)$ as obtained for the MEM. At high temperatures $P_{L}(m)$ is a Gaussian centered at $m=0$ but when the temperature gets lowered, the distribution broadens and develops two peaks at $m=1$ and $m=-1$. Further decreasing the temperature causes these peaks to become dominant while the distribution turns distinctly non-Gaussian, exhibiting a minimum just at $m=0$. The emergence of the maxima at $m$ $= \pm 1$ is quite abrupt. This behavior reminds us the order parameter probability distribution characteristic of the onedimensional Ising model. In fact, for the well-studied $d$-dimensional Ising model $[32,36]$, we know that for $T$ $>T_{c}, P_{L}(M)$ is a Gaussian centered at $M=0$, given by

$$
P_{L}(M) \propto \exp \left(\frac{-M^{2} L^{d}}{2 T \chi}\right),
$$

where the susceptibility $\chi$ is related to order parameter fluctuations by

$$
\chi=\frac{L^{d}}{T}\left(\left\langle M^{2}\right\rangle-\langle M\rangle^{2}\right) .
$$

Decreasing temperature, the order parameter probability distribution broadens, it becomes non-Gaussian, and near $T_{c}$ it splits into two peaks that get more separated at lower temperature. For $T<T_{c}$ and linear dimensions $L$ much larger than the correlation length $\xi$ of order parameter fluctuations, one may approximate $P_{L}(M)$ near the peaks by a doubleGaussian distribution, i.e.,

$$
P_{L}(M) \propto \exp \left[\frac{-\left(M-M_{s p}\right)^{2} L^{d}}{2 T \chi}\right]+\exp \left[\frac{-\left(M+M_{s p}\right)^{2} L^{d}}{2 T \chi}\right]
$$

where $M_{s p}$ is the spontaneous magnetization, while the susceptibility $\chi$ is now given by

$$
\chi=\frac{L^{d}}{T}\left(\left\langle M^{2}\right\rangle-\langle|M|\rangle^{2}\right) .
$$

From Eq. (4), it turns out that the Gaussian squared width $\sigma^{2}$ associated with high temperature distributions is very close to the second moment of the order parameter, i.e.,

$$
\sigma^{2} \approx\left\langle M^{2}\right\rangle .
$$

Equation (8) is a consequence of the Gaussian shape of the order parameter probability distribution and, thus, it holds for the MEM as well. From the known one-dimensional exact solution for a chain of $L$ spins $[37,38]$ one can obtain

$$
\chi=\frac{1}{T} \exp (2 / T)
$$

then, Eqs. (5) and (9) lead us to

$$
\left\langle M^{2}\right\rangle=\frac{1}{L} \exp (2 / T)
$$

(where it has been taken into account that $\langle M\rangle=0$ due to finite-size effects, irrespective of temperature). From Eqs. (8) and (10) we can see that the high temperature Gaussian probability distribution broadens exponentially as $T$ gets lowered, until it develops deltalike peaks at $M= \pm 1$ as a consequence of a boundary effect on the widely extended distribution. It should be noticed that for $d \geqslant 2$ this phenomenon is prevented by the finite critical temperature that splits the Gaussian, as implied by Eq. (6).

Turning back to the MEM, Fig. 1 strongly suggests that an analogous mechanism should be responsible for the thermal dependence exhibited by the MEM's order parameter distribution function. So, by analogy to Eq. (9), we assume the relation

$$
\chi=\frac{1}{T} \exp (a / T)
$$

to hold for the MEM, where we have introduced a phenomenological parameter $a$, and the susceptibility $\chi$ is given by Eq. (5). We find an excellent agreement to the data by choosing the value $a=1.6$ as observed in Fig. 2(a), where ln-linear plots of $L\left\langle m^{2}\right\rangle$ versus $1 / T$ are shown for strip widths varying 

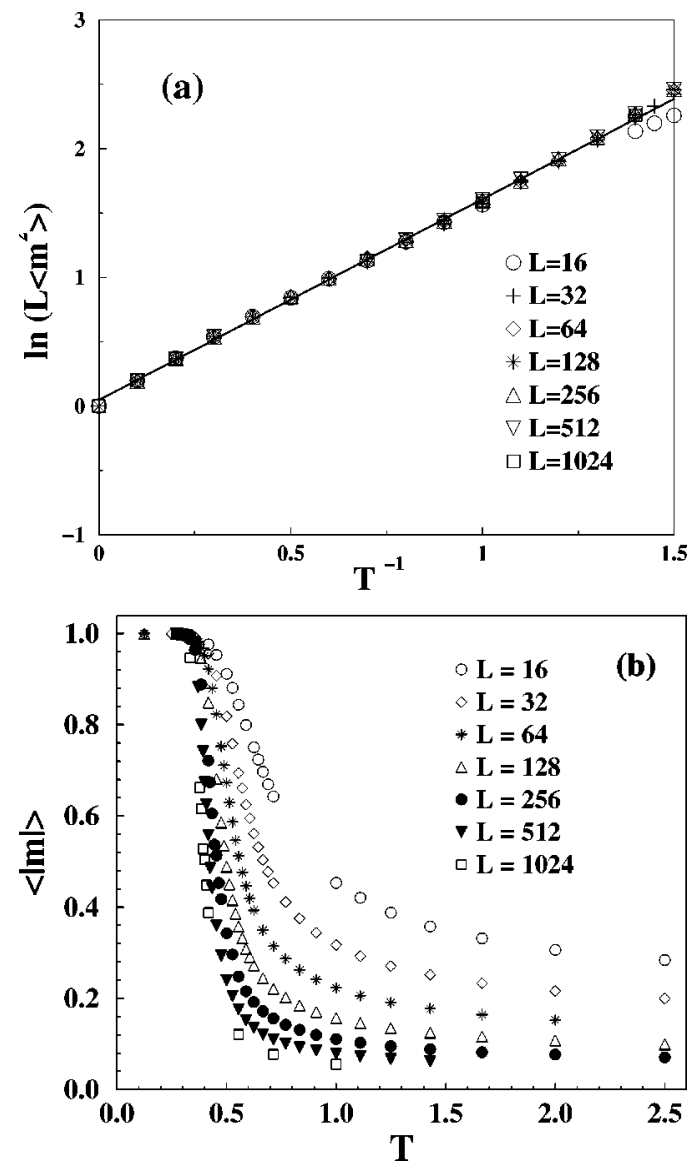

FIG. 2. Data for strip widths in the range $16 \leqslant L \leqslant 1024$. (a) Log-linear plots of $L\left\langle m^{2}\right\rangle$ versus $T^{-1}$. The slope of the solid line (linear fit to the data) is $a=1.6$. (b) Plots of $\langle|m|\rangle$ versus $T$. More details in the text.

in the range $16 \leqslant L \leqslant 1024$. Figure 2 (b) shows plots of $\langle|m|\rangle$ versus $T$ for the same lattices. This figure shows that by increasing $L$, the order parameter curves approach the one that corresponds to the thermodynamic limit (i.e., $\langle|m|\rangle$ $=\theta(T)$, where $\theta$ is the Heaviside function).

However, it should be pointed out that the results obtained for the $(1+1)$-dimensional MEM and the one-dimensional (1D) Ising model do not exactly coincide for finite lattices, as Fig. 3(a) shows for the case of the magnetization. Anyway, this fact should not alarm us, since it can be seen that differences in the results obtained for both models are a direct consequence of the finite-size nature of the lattices used in the simulations and consequently they tend to vanish in the thermodynamic limit. This is actually shown by Fig. 3(b), where log-linear plots of $\left\langle|M|_{\text {Ising }}\right\rangle(L, T)-\left\langle|m|_{M E M}\right\rangle(L, T)$ versus $L^{-1}$ for two different fixed values of temperature are presented. Thus, we conclude that in view of the full qualitative and quantitative agreement between both models we can safely establish that, as in the 1d Ising model, the (1 +1 )-dimensional MEM is not critical (i.e., it also undergoes a phase transition at $T_{c}=0$ ).

We have also computed the number of already occupied NN sites every time a new particle was added to the spin system, and thus we have obtained the normalized probability $P\left(n_{N N}\right)$ of having $n_{N N}$ occupied NN sites. Figure 4 shows
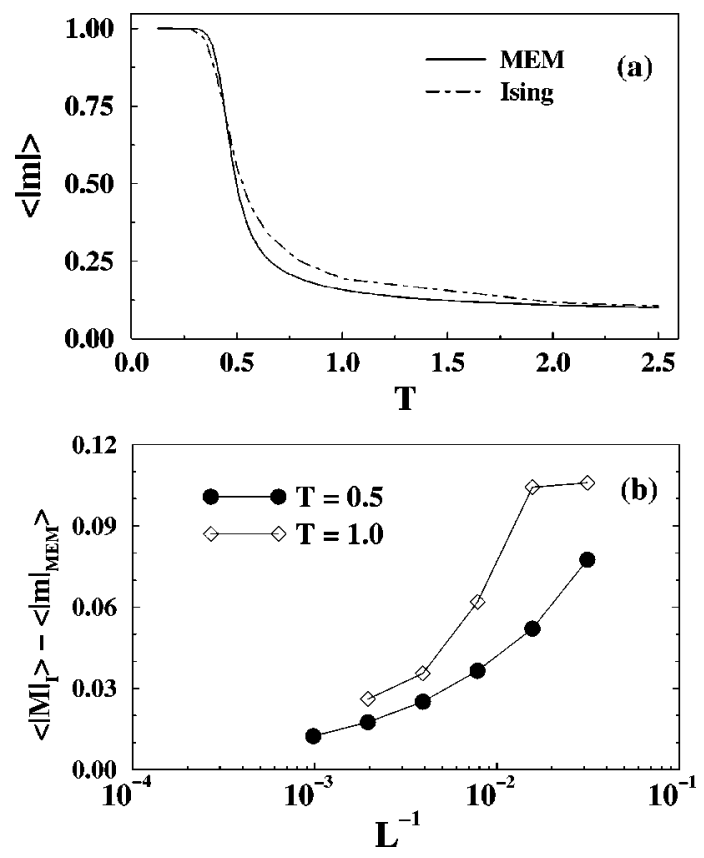

FIG. 3. Comparison of results corresponding to the $(1+1)$-dimensional MEM and the $d=1$ Ising model. (a) Plots of $\langle|m|\rangle$ versus $T$ obtained for a lattice of side $L=128$. (b) Linear-log plots of $\left\langle|M|_{I}\right\rangle(L, T)-\left\langle|m|_{M E M}\right\rangle(L, T)$ versus $L^{-1}$ for $T=0.5$ and $T=1.0$. Hence, differences in the magnetization due to finite-size effects appear to vanish in the thermodynamic limit.

the behavior of $P\left(n_{N N}\right)$ as a function of temperature. Using this probability we have evaluated $\left\langle n_{N N}\right\rangle=2.0000(1)$ irrespective of the temperature. This result can be understood considering that the growing process that leads to the assignment of a spin $S_{i j}= \pm 1$ to each lattice site of coordinates $(i, j)$ can be studied by means of a bond model. In fact, we can assign a bond to each pair of neighboring sites, pointing from the earlier occupied site to the later occupied one. So, the process that leads to a given spin configuration can be specified by the fields $b_{U}(i, j)$ and $b_{R}(i, j)$, where subindexes $U$ and $R$ refer to the upper bond of $(i, j)$ [i.e., the bond that connects the site of coordinates $(i, j)$ with that of coordinates $(i, j+1)]$, and to the right bond of the site of coordinates $(i, j)$, respectively [39]. We take $b(i, j)=+1$ if the

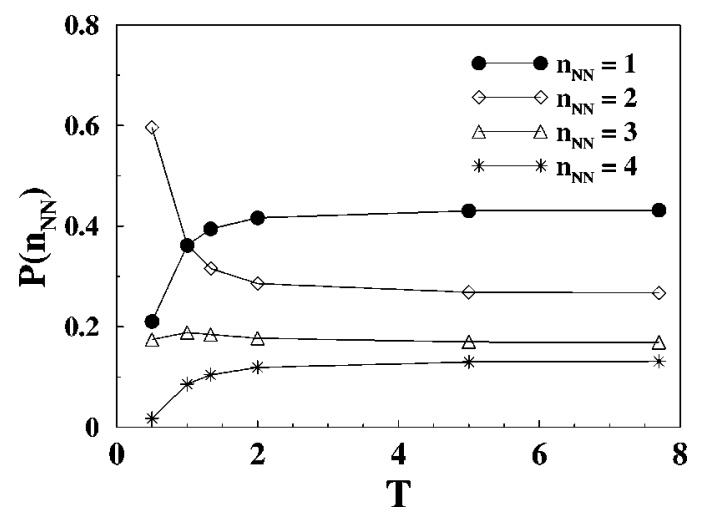

FIG. 4. Plots of $P\left(n_{N N}\right)$ versus $T$ for $n_{N N}=1,2,3,4$. The lines are guides to the eye. More details in the text. 


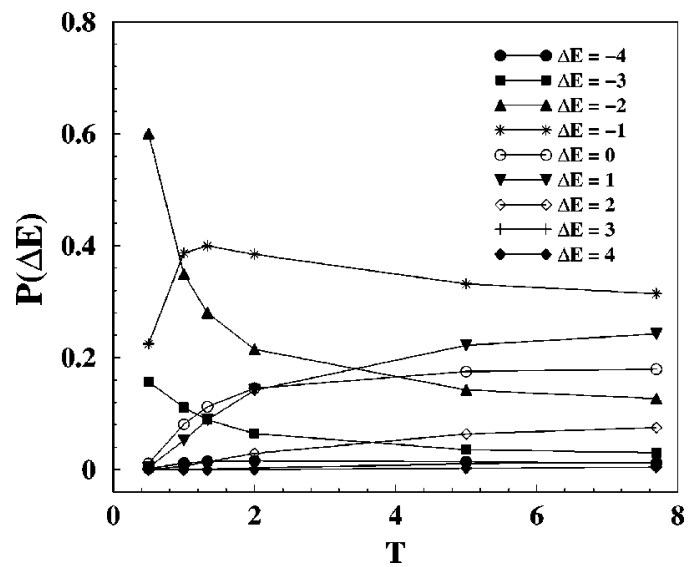

FIG. 5. Plots of $P(\Delta E)$ versus $T$ for $\Delta E=0, \pm 1, \pm 2, \pm 3, \pm 4$ (in units of $J$ ). The lines are guides to the eye. More details in the text.

bond points outwards and $b(i, j)=-1$ if it is directed inwards. Therefore, the net bond flux at a given lattice site $(i, j)$ is given by

$$
\phi(i, j)=b_{U}(i, j)+b_{R}(i, j)-b_{U}(i, j-1)-b_{R}(i-1, j)
$$

and the possible values that $\phi$ can take are $\phi=-4,-2,0,2$. After some algebra, it follows that $n_{N N}=\frac{1}{2}(4-\phi)$ holds for every site on the lattice. Moreover, it can be seen that, for an arbitrary $d$-dimensional lattice of coordination number $q$, $n_{N N}=\frac{1}{2}(q-\phi)$. Then,

$$
\left\langle n_{N N}\right\rangle=\frac{1}{2} q
$$

is the mean number of occupied NN, since $\langle\phi\rangle=0$. For the two-dimensional square lattice, $q=4$ and Eq. (13) yields $\left\langle n_{N N}\right\rangle=2$, in agreement with the result we have already obtained by means of Monte Carlo simulations.

Further insights into the MEM's growing process can be gained by studying the mean energy change involved in the addition of a new particle to the system. The process of adding a new spin involves an energy change $\Delta E$ and from the definition of the $(1+1)$-dimensional MEM, the possible values that $\Delta E$ can take are $0, \pm 1, \pm 2, \pm 3, \pm 4$ (in units of $J$ ). Figure 5 shows plots of the normalized probability $P(\Delta E)$ versus $T$ for each of these values. The nonequilibrium nature of the MEM manifests itself through much more complex probability distributions $P(\Delta E)$ (see Fig. 5) than those corresponding to the equilibrium 1D Ising model where $\Delta E$ can take only three different values, namely, $0, \pm 4$ (in units of $J$ ). The results shown in Figs. 4 and 5 confirm the nontrivial nature of the link established between the MEM at stationarity and the Ising model in equilibrium.

It should be noticed that for the case of CGM [22-24] the growing conditions are quite different than those of the MEM. In fact, in CGM the crystal grows layer by layer in a given direction $[24,26]$. Furthermore, the probability distribution of the predecessor spin layer is sampled from the equilibrium distribution, so will be the probability of spins in subsequent layers. This particular growth mechanism allows

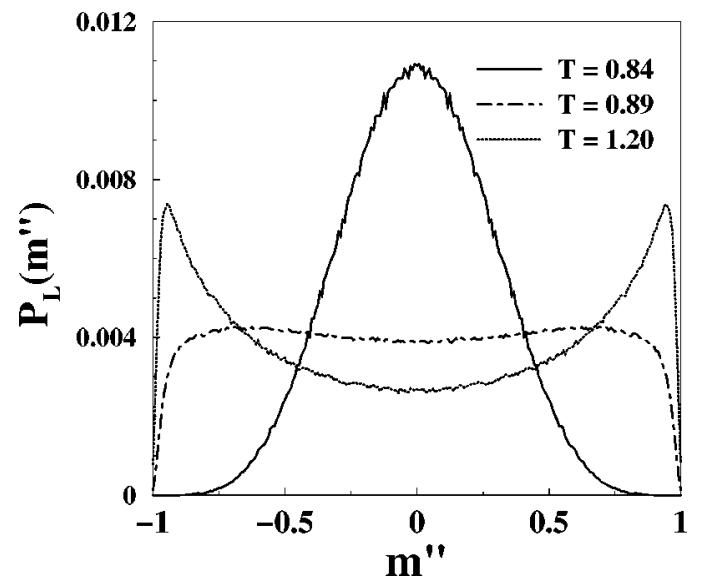

FIG. 6. Plots of the probability distribution $P_{L}\left(m^{\prime \prime}\right)$ versus $m^{\prime \prime}$ for the fixed lattice size $L=16$ and different temperatures. The occurrence of two maxima located at $m^{\prime \prime}= \pm M_{s p}$ (for a given value of $M_{s p}$ such that $0<M_{s p}<1$ ) is the hallmark of a thermal continuous phase transition that takes place at a finite critical temperature.

to establish dual transformations with the kinetic Ising model $[25,26]$ and to extract some exact results. In contrast, the growing interface of the MEM is self-affine and the system is far from equilibrium. So, the link between the 1D Ising model and the $(1+1)$-dimensional MEM is quite challenging.

\section{STUDY OF THE MEM IN (2+1)-DIMENSIONS: RESULTS AND DISCUSSION}

\section{A. The order parameter and its probability distribution function}

In order to compare the $(2+1)$-dimensional MEM and the 2D Ising model, we have first studied the order parameter probability distribution $P_{L}\left(m^{\prime \prime}\right)$, where $m^{\prime \prime}$ takes now $L^{2}$ +1 possible values (see Fig. 6). For high temperatures, the probability distribution corresponds to a Gaussian centered at $m^{\prime \prime}=0$. At lower temperatures we observe the onset of two maxima located at $m^{\prime \prime}= \pm M_{s p}\left(0<M_{s p}<1\right)$, which become sharper and approach $m^{\prime \prime}= \pm 1$ as $T$ is gradually decreased. Figure 7 shows the location of the maximum of the probability distribution as a function of temperature for both $(d$ +1 )-dimensional MEM models with $d=1,2$. While for the $d=2$ case we observe a smooth transition from the $m_{\max }^{\prime \prime}$ $=0$ value characteristic of high temperatures to nonzero $m_{\max }^{\prime \prime}$ values that correspond to lower temperatures, the curve obtained for $d=1$ shows, in contrast, a Heaviside-like jump. As already discussed, the behavior exhibited by the $(2+1)$-dimensional MEM (e.g., as displayed by Figs. 6 and 7) is the signature of a thermal continuous phase transition that takes place at a finite critical temperature.

The broken symmetry at a finite critical temperature $T_{c}$ implied by the thermal continuous phase transition can be explained in terms of the broken ergodicity that occurs in the system when we tend to the thermodynamic limit $(L \rightarrow \infty)$ making use of the temperature dependence exhibited by the order parameter distribution function. In fact, if we set the characteristic length of MEM's domains $l_{D}$ equal to an er- 


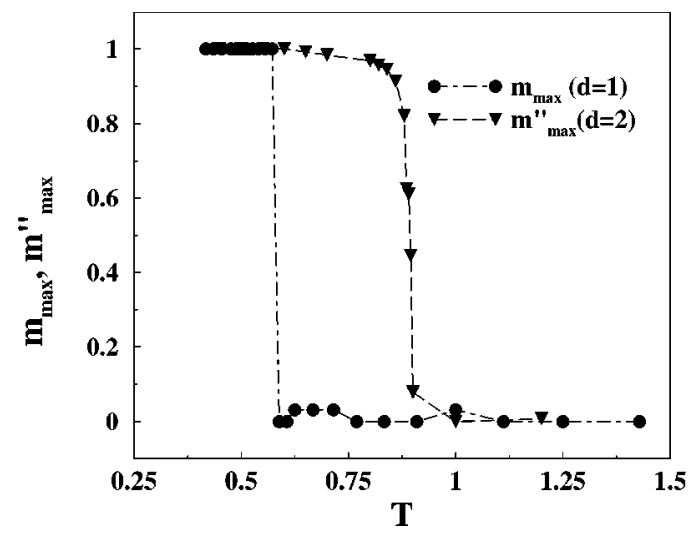

FIG. 7. Plots showing the location of the maximum of the probability distribution as a function of temperature for both $(d+1)$-dimensional MEM models $(d=1,2)$. The lines are guides to the eye. The smooth transition for $d=2$ constitutes another evidence of the finite critical point associated with the $(2+1)$-dimensional MEM.

godic length $l_{\text {erg }}$, we can carry out a complete analogy with the Ising model by associating $l_{\text {erg }}$ to $t_{\text {erg }}$ (the Ising model ergodic time) and the cluster's total length $l_{C}$ (already defined in Sec. III) to the Ising model observation time $t_{o b s}$. In this way, we encounter that excursions of $m^{\prime \prime}$ from $m^{\prime \prime}$ $=+M_{s p}$ to $m^{\prime \prime}=-M_{s p}$ and vice versa occur at length scales of the order of $l_{\text {erg }}$. When the cluster's total length becomes larger and larger $\left(l_{C} \gg l_{\text {erg }}\right)$ the whole cluster's magnetization is averaged to zero. Furthermore, $l_{\text {erg }}$ diverges as the strip's width becomes larger and larger, and again broken symmetry arises as the consequence of broken ergodicity.

\section{B. Order-disorder phase transition in the $(2+1)$-dimensional MEM: Finite-size effects and scaling analysis}

As already anticipated and as it follows from Figs. 6 and 7, the $(2+1)$-dimensional MEM exhibits a thermally driven order-disorder transition at a finite temperature. In the thermodynamic limit $(L \rightarrow \infty)$ we expect to determine a critical temperature $T_{c}$ such that $\left\langle\left|m^{\prime \prime}\right|\right\rangle=0$ for $T>T_{c}$ while $\left\langle\left|m^{\prime \prime}\right|\right\rangle$ remains nonvanishing at temperatures below $T_{c}$.

From the finite-size scaling theory, developed for the treatment of finite-size effects at criticality and under equilibrium conditions [40,41], it is well known that if a thermally driven phase transition occurs at a temperature $T_{c}>0$ in the thermodynamic limit, then in a confined geometry this transition becomes smeared out over the temperature region $\Delta T(L)$ around a shifted effective transition temperature $T_{c}(L)$, and the following relationships hold:

$$
\Delta T(L) \propto L^{-\theta},
$$

and

$$
\left|T_{c}(L)-T_{c}\right| \propto L^{-\lambda}
$$

where the rounding and shift exponents are given by $\theta=\lambda$ $=\nu^{-1}$, respectively (recalling that $\nu$ is the exponent that characterizes the divergence of the correlation length at criticality).

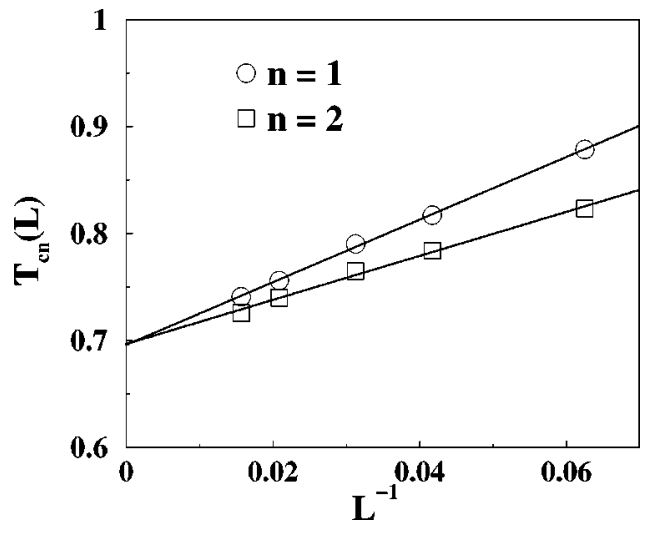

FIG. 8. Plots of $T_{c n}(L)$ versus $L^{-1}$ (for $n=1,2$ ). The solid lines show the linear extrapolations that meet at the critical point given by $T_{c}=0.69 \pm 0.01$.

Furthermore, from well-established finite-size scaling relations, the following Ansätze hold just at criticality:

$$
\left\langle\left|m^{\prime \prime}\left(L, T=T_{c}\right)\right|\right\rangle \propto L^{-\beta / \nu}
$$

and

$$
\chi_{\max }(L) \propto L^{\gamma / \nu}
$$

where $\beta$ and $\gamma$ are the order parameter and the susceptibility critical exponents, respectively. Note that $\chi_{\max }(L)$, as given by Eq. (17), refers to the maximum of $\chi(L, T)$ as a function of $T$ for fixed lattice size $L$.

In view of the encountered analogies between the MEM and the Ising model, it is natural to test the validity of Eqs. (14)-(17) for the case of the MEM in $(2+1)$ dimensions. It should be noted that as in the case of equilibrium systems, in the present case various "effective", $L$-dependent critical temperatures can also be defined. In particular, we will define $T_{c 1}(L)$ as the value that corresponds to $\left\langle\left|m^{\prime \prime}\right|\right\rangle=0.5$ for fixed $L$, and $T_{c 2}(L)$ as the one corresponding to the maximum of the susceptibility for a given $L$, assuming that the susceptibility is related to order parameter fluctuations in the same manner as for equilibrium systems [as given by Eqs. (5) and (7)]. Then, we should be able to obtain $T_{c}$ from plots of $T_{c n}(L)$ versus $L^{-1}$ (for $n=1,2$ ) as is shown in Fig. 8. Following this procedure we find that both $T_{c 1}(L)$ and $T_{c 2}(L)$ extrapolate (approximately) to the same value, allowing us to evaluate the critical temperature $T_{c}=0.69 \pm 0.01$ in the thermodynamic limit.

After determining $T_{c}$, the correlation length exponent $\nu$ can be evaluated by means of Eq. (15), making the replacement $\lambda=1 / \nu$. In fact, taking $T_{c}$ at the mean, maximum, and minimum values allowed by the error bars, we obtain six $\log$ - $\log$ plots of $\left|T_{c n}(L)-T_{c}\right|$ versus $L$ for $n=1,2$. The slope of each of these plots, not shown here for the sake of space, yields a value for $\nu$. The obtained values are

$$
\begin{gathered}
\nu=1.08\left(T_{c}=0.68\right), \quad \nu=1.00\left(T_{c}=0.69\right), \\
\nu=0.88\left(T_{c}=0.70\right) \quad \text { for } n=1,
\end{gathered}
$$




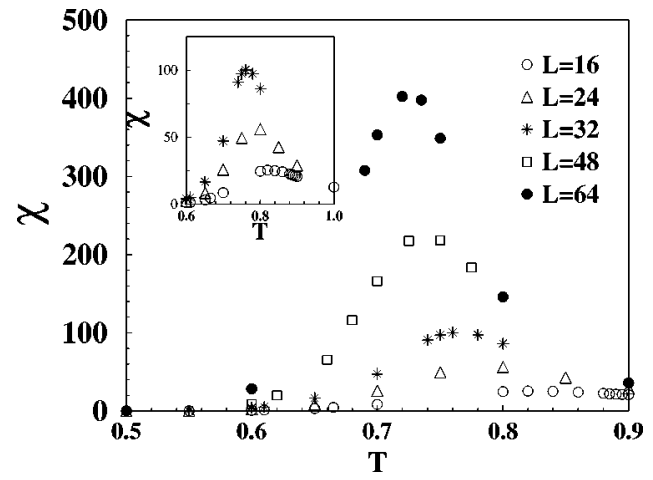

FIG. 9. Behavior of the susceptibility as a function of temperature. Each curve shows a peak that becomes sharper and shifts towards lower temperatures as $L$ is increased. The inset shows in greater detail the peaks corresponding to the smaller lattices ( $L$ $=16,24,32)$.

and

$$
\begin{gathered}
\nu=1.20\left(T_{c}=0.68\right), \quad \nu=1.08\left(T_{c}=0.69\right), \\
\nu=0.95\left(T_{c}=0.70\right) \quad \text { for } n=2 .
\end{gathered}
$$

Thus our estimate is given by $\nu=1.04 \pm 0.16$, where the error bars reflect the error derived from the evaluation of $T_{c}$, as well as the statistical error.

Figure 9 shows plots of the susceptibility versus $T$ as obtained using lattices of different side. It is found that the susceptibility exhibits a peak that becomes sharper and shifts towards lower temperatures when $L$ is increased. This behavior is, in fact, already anticipated by Eq. (17), and it allows us to evaluate $\gamma / \nu$ from the slope of a log-log plot of $\chi_{\max }$ versus $L$, as Fig. 10 shows. The linear fit yields $\gamma / \nu=2.02$ \pm 0.04 . Using this value and the value formerly obtained for $\nu$ we thus determine $\gamma=2.10 \pm 0.36$.

Figure 11 shows log-log plots of $\left\langle\left|m^{\prime \prime}\right|\right\rangle\left(T=T_{c}\right)$ versus $L$ for the mean, maximum, and minimum allowed values of $T_{c}$. Considering only the larger lattices, the linear fits to the data according to Eq. (16) yield the following estimates: $\beta / \nu=0.11, \beta / \nu=0.16$, and $\beta / \nu=0.19$. We then assume the value $\beta / \nu=0.15 \pm 0.04$, where the error bars reflect the error derived from the evaluation of $T_{c}$ as well as the statistical

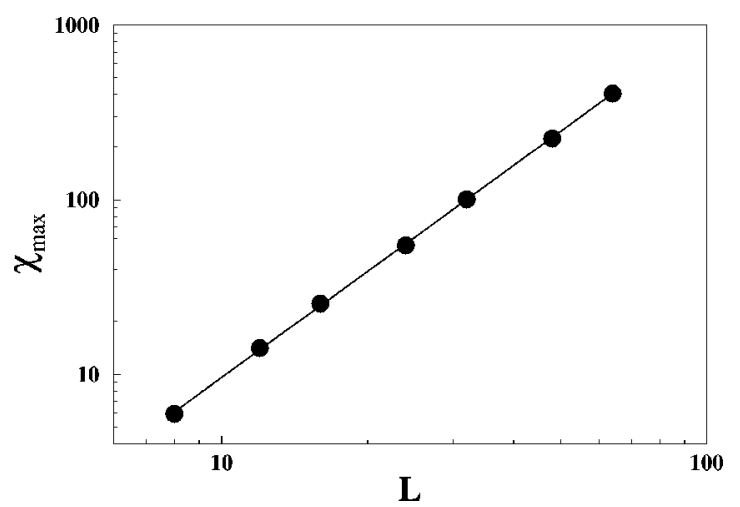

FIG. 10. Log-log plot of $\chi_{\max }$ versus $L$. The linear fit (solid line) yields $\gamma / \nu=2.02 \pm 0.04$.

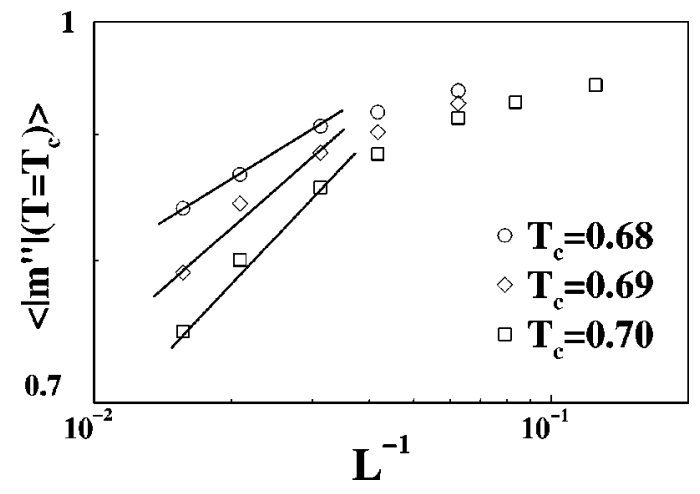

FIG. 11. Log-log plots of $\left\langle m^{\prime \prime}\left(T=T_{c}\right)\right\rangle$ versus $L^{-1}$ for the mean, maximum, and minimum allowed values of $T_{c}$. The linear fits (solid lines) yield an estimate $\beta / \nu=0.16 \pm 0.05$.

error. From this value and the value formerly obtained for $\nu$ we thus determine $\beta=0.16 \pm 0.05$.

The critical exponents of the MEM in $(2+1)$ dimensions as obtained using a finite-size scaling analysis are so far: $\nu$ $=1.04 \pm 0.16, \gamma=2.10 \pm 0.36$, and $\beta=0.16 \pm 0.05$. If we recall the exactly known critical exponents of the $d=2$ Ising model, i.e. $\nu=1, \gamma=7 / 4$, and $\beta=1 / 8$, we find that the ( 2 $+1)$-dimensional MEM has the same critical exponents within error bars. These results further support our conjecture on the connection between the MEM in $(2+1)$ dimensions and the Ising model in two dimensions.

As in the case of the MEM in $d=1$, we have also computed the number of already occupied NN sites every time that a new particle was added to the spin system. We found that the value $\left\langle n_{N N}\right\rangle=3.0000$ (1) holds for all temperatures, which is indeed the result given by Eq. (13), since $q=6$ for the three-dimensional square lattice.

At this stage, we may recall that for the $(1+1)$-dimensional MEM $\left\langle n_{N N}\right\rangle$ equals the coordination number of the $d=1$ Ising model, and that we found that both models have the same critical temperature and exhibit the same critical behavior. Reasoning by analogy, we may expect a coincidence between the critical temperature for the $(2+1)$-dimensional MEM and the corresponding one for a $d=2$ Ising model defined on a lattice of coordination number $q=3$. However, this comparison cannot be carried out, since the critical temperature of an Ising model depends on both the coordination number $q$ and the topological structure of the lattice, but for $d \geqslant 2$ and a given value of $q$ the topological structure is not unique. For instance, for $d=2$ and $q=3$, we can pass from the honeycomb lattice (HL) to the expanded kagomé lattice (EKL) through the application of a star-triangle transformation and obtain the exact values of their critical points, which turn out to be [42] $T_{c}=1.5187$ $(\mathrm{HL})$ and $T_{c}=1.4530(\mathrm{EKL})$.

\section{CONCLUSIONS}

In the present work we have studied the growth of magnetic Eden clusters with ferromagnetic interactions between nearest-neighbor spins in a $(d+1)$-dimensional rectangular geometry (for $d=1,2$ ), using Monte Carlo simulations and 
applying a finite-size scaling theory. The results obtained allow us to conjecture a nontrivial correspondence between the MEM for the irreversible growth of magnetic materials and the classical Ising model under equilibrium conditions. In fact, we have found that the $(d+1)$-dimensional MEM and the $d$-dimensional Ising model behave identically (except for finite-size differences that vanish in the thermodynamic limit) at criticality, i.e., that both models belong to the same universality class. We also conjecture that this correspondence would remain at higher dimensions $(d>2)$. The results obtained strongly suggest a link between the temporal evolution of equilibrium systems and the stationary growth of nonequilibrium systems. We thus believe that this work will stimulate further developments in the field of nonequilibrium kinetic growth models. A more precise numerical test of the posed conjecture will certainly require a considerable computational effort but it will be of great interest. Furthermore, analytical developments aimed to establish a theoretical framework for the understanding of far from equilibrium growth phenomena will become stimulated by the reported findings.

\section{ACKNOWLEDGMENTS}

This work is supported financially by CONICET, UNLP, CIC (Bs. As.), ANPCyT, and Fundación Antorchas (Argentina), and the Volkswagen Foundation (Germany). The authors thank M. Muñoz for helpful discussions and his proposal of the bond representation for the MEM.
[1] F. Family and T. Vicsek, Dynamics of Fractal Surfaces (World Scientific, Singapore, 1991).

[2] A. L. Barabasi and H. E. Stanley, Fractal Concepts in Surface Growth (Cambridge University Press, New York, 1995).

[3] Fractals and Disordered Media, edited by A. Bunde and S. Havlin (Springer-Verlag, Heidelberg, 1991).

[4] Fractals in Science, edited by A. Bunde and S. Havlin (Springer-Verlag, Heidelberg, 1995).

[5] M. Marsili, A. Maritan, F. Toigo, and J. R. Banavar, Rev. Mod. Phys. 68, 963 (1996).

[6] M. Eden, in Symposium on Information Theory in Biology, edited by H. P. Yockey (Pergamon Press, New York, 1958); in Proceedings of the Fourth Berkeley Symposium on Mathematics, Statistics and Probability, edited by F. Neyman (University of California Press, Berkeley, 1961), Vol. IV, p. 223.

[7] D. E. Wolf and J. Kertész, Europhys. Lett. 4, 651 (1987).

[8] M. Cieplak, A. Maritan, and J. R. Banavar, Phys. Rev. Lett. 76, 3754 (1996).

[9] C. S. Ryu and In-mook Kim, Phys. Rev. E 53, 5643 (1996).

[10] S. S. Manna and D. Dhar, Phys. Rev. E 54, R3063 (1996).

[11] E. V. Albano, Phys. Rev. E 56, 7301 (1997).

[12] M. Marsili and M. Vendruscolo, Europhys. Lett. 37, 505 (1997).

[13] M. Ausloos, N. Vandewalle, and R. Cloots, Europhys. Lett. 24, 629 (1993); N. Vandewalle and M. Ausloos, Phys. Rev. E 50, R635 (1994).

[14] Y. V. Ivanenko, N. I. Lebovka, and N. V. Vygornitskii, Eur. Phys. J. B 11, 469 (1999).

[15] E. Ising, Z. Phys. 31, 253 (1925).

[16] J. Shen et al., Phys. Rev. B 56, 2340 (1997).

[17] O. Pietzsch, A. Kubetzka, M. Bode, and R. Wiesendanger, Phys. Rev. Lett. 84, 5212 (2000).

[18] J. Hauschild, U. Gradmann, and H. J. Elmers, Appl. Phys. Lett. 72, 3211 (1998).

[19] H. J. Elmers, J. Hauschild, and U. Gradmann, Phys. Rev. B 59, 3688 (1999).

[20] U. Bovensiepen et al., Phys. Rev. Lett. 81, 2368 (1998).

[21] B. M. McCoy and T. T. Wu, The Two Dimensional Ising Model (Harvard University Press, Cambridge, MA, 1973).
[22] T. R. Welberry and R. Galbraith, J. Appl. Crystallogr. 6, 87 (1973).

[23] A. M. Verhagen, J. Stat. Phys. 15, 219 (1976).

[24] I. G. Enting, J. Phys. C 10, 1379 (1977).

[25] P. Ruján, J. Stat. Phys. 29, 231 (1982).

[26] P. Ruján, J. Stat. Phys. 34, 615 (1984).

[27] P. Ruján, J. Stat. Phys. 49, 139 (1987).

[28] J. Stephenson, Phys. Rev. B 1, 4405 (1970); J. Math. Phys. 11, 420 (1970).

[29] K. Binder and D. W. Heermann, Monte Carlo Simulation in Statistical Physics, Springer Series in Solid State Sciences Vol. 80 (Springer-Verlag, Berlin, 1992).

[30] K. Binder and D. Stauffer, in Applications of the Monte Carlo Method in Statistical Physics, 2nd ed., edited by K. Binder (Springer-Verlag, Berlin, 1987).

[31] K. Binder, in Encyclopedia of Applied Physics (VCH Publishers, Heidelberg, 1994), Vol. 10, p. 567.

[32] K. Binder, in Monte Carlo and Molecular Dynamics of Condensed Matter Systems, Conference Proceedings Vol. 49, edited by K. Binder and G. Ciccotti (SIF, Bologna, 1996), Chap. 5, p. 124.

[33] K. Binder, Z. Phys. B: Condens. Matter 43, 119 (1981).

[34] A. D. Bruce, J. Phys. C 14, 3667 (1981).

[35] M. M. Tsypin and H. W. J. Blöte, Phys. Rev. E 62, 73 (2000).

[36] D. P. Landau and E. M. Lifshitz, Statistical Physics, 3rd ed. (Pergamon Press, Oxford, 1980), Part 1.

[37] R. J. Baxter, Exactly Solved Models in Statistical Mechanics (Academic Press, New York, 1982).

[38] K. Huang, Statistical Mechanics, 2nd ed. (John Wiley, New York, 1987), p. 363.

[39] Since we are dealing with periodic boundary conditions in the $j$ direction, we consider $j-1=L$ if $j=1$ and analogously $j+1=1$ if $j=L$ throughout.

[40] M. N. Barber, in Phase Transitions and Critical Phenomena, edited by C. Domb and J. L. Lebowitz (Academic, New York, 1983), Vol. 8, p. 146.

[41] Finite Size Scaling and Numerical Simulations of Statistical Systems, edited by V. Privman (World Scientific, Singapore, 1990).

[42] M. Fisher, Phys. Rev. 113, 969 (1959). 